\title{
An Online Degradation Feature Extraction Technique for Shore Bridge Gearbox Based on Morphological Fractal Dimension and Sliding Window Weibull Fitting
}

\author{
Dejian Sun, Bing Wang $(\mathbb{D}$, Xiong Hu, and Wei Wang \\ Shanghai Maritime University, Shanghai 201306, China \\ Correspondence should be addressed to Bing Wang; 1002624905@qq.com
}

Received 22 April 2019; Accepted 17 September 2019; Published 30 October 2019

Guest Editor: Franco Concli

Copyright @ 2019 Dejian Sun et al. This is an open access article distributed under the Creative Commons Attribution License, which permits unrestricted use, distribution, and reproduction in any medium, provided the original work is properly cited.

\begin{abstract}
Shore bridge and other port cranes have some working condition characters including high speed, heavy load, and large impact. In order to solve the degradation feature extraction issue of hoisting mechanism gearbox, an online degradation feature extraction technique based on morphological fractal dimension and sliding window Weibull fitting is proposed. Firstly, taking the vibration energy spectrum collecting from the gearbox as the online data source, the fractal dimension of the vibration energy spectrum during an analysis period is calculated and a fractal evolution curve is obtained. A three-parameter Weibull fitting on the fractal curve within a sliding window after setting the window's width and step size is performed. The scale parameter of the Weibull fitting model is introduced as the performance degradation feature. The effectiveness of the technique is verified by the full-life vibration data of hoisting gearbox from Shanghai Port Group. The results show that the morphological fractal dimension is able to describe the fractal complexity of the vibration energy spectrum. The scale parameter of Weibull distribution is able to reflect the performance degradation trend of fractal curve smoothly, which lays a theoretical foundation for further solving the problem of online health assessment.
\end{abstract}

\section{Introduction}

Shore bridge is an important container lifting equipment in the harbor that has a harsh operating environment and special working condition. The hoisting mechanism is the key system in shore bridge for container lifting operations which is shown in Figure 1. In the process of high-speed, heavy-load, and large-impact periodic container-hoisting operations, the gearbox will withstand vibration shock caused by strong dynamic loading, which will induce performance degradation or even sudden failure under longterm action [1], resulting in production stoppage or even casualties. If degradation features can be monitored and extracted online, the performance degradation trend of gearbox will be accurately tracked and health condition is able to be accurately assessed to improve the safety and reliability of the hoisting mechanism in the harbor.

The purpose of degradation feature extraction is excavating degradation pattern involved in signals quantitatively, which will construct the basis of health condition assessment. Linear signal processing methods such as time-domain, frequency-domain, and time-frequency-domain analysis are usually introduced and applied such as kurtosis [2], spectral kurtosis [3], and adaptive modal decomposition $[4,5]$. Considering the nonlinear and nonstationary character of mechanical vibration signals, complexity analysis methods based on information entropy and fractal theory have been applied to degradation feature analysis of rotating machinery such as bearing and gearbox in recent years, including approximate entropy, fuzzy entropy, sample entropy, box dimension, correlation dimension, and other features [6-10]. Bearing full-life acceleration test data from IMS [11] and IEEE PHM 2012 [12] are usually used in methods verifying due to the advantages of small load disturbance and obvious degradation pattern. Compared with the accelerated test data, vibration signal of the shore bridge in industrial site is more complicated. The special working condition will induce intensive vibration, and 


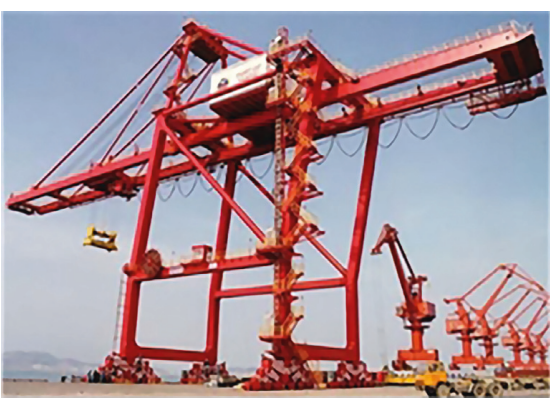

(a)

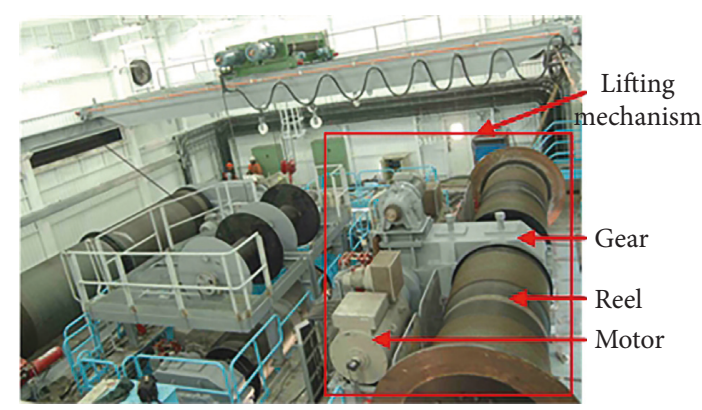

(b)

Figure 1: (a) Real map of quay crane and (b) its lifting mechanism.

complex industrial environment will introduce a large amount of noise and random impact; the combination of these factors bring the vibration signal nonlinear, nonstationary, and aperiodic characters, so that it is hard to exploit the performance degradation pattern with traditional methods effectively. In addition, the shore bridge has a long design life, so it is very difficult to achieve a long-term online monitoring to verify these techniques. The above difficulties in techniques and verifying have constrained the development of PHM technologies in port cranes. It is of great significance to study the effective degradation feature extraction method and excavate the degradation pattern in complex industrial field data to achieve accurate health condition assessment for shore bridge and other port cranes.

Preliminary study has shown that the vibration energy spectrum of shore bridge has certain self-similarity and longrange correlation which indicate a fractal character of the vibration energy spectrum. In the complexity analysis method, fractal dimension is able to describe complexity quantitatively and some studies have been processed in fault feature extraction $[13,14]$ as well as degradation feature extraction [15]. Fractal dimension calculated by mathematical morphological theory is able to overcome the shortcomings of traditional boxcounting dimension in accuracy and has some advantages of stability, accuracy, and calculating speed. Therefore, it is feasible to analyze fractal dimension variation for vibration energy spectrum during the degradation process. In addition, Weibull distribution is able to describe life distribution of mechanical equipment and components, while three-parameter Weibull distribution is more accurate due to the introduction of positional parameters compared with two-parameter Weibull distribution and has been successfully used in fault diagnosis $[16,17]$. For filtering the numerical fluctuation caused by random shock caused by strong dynamic loading, Weibull distribution is able to explore the stability variation pattern from the perspective of statistical distribution. In view of the method verification, the NetCMAS system which has achieved industry 4.0 level 4 standard is able to provide a reliable platform for data acquisition. At present, multiple sets of fulllife vibration energy spectrums for port crane components have been monitored and stored [18], which could provide a reliable data support for industrial applied research.

In summary, an online degradation feature extraction technique based on morphological fractal dimension and sliding window Weibull fitting is proposed. The paper is organized as follows: Section 2 briefly introduces mathematical morphological fractal dimension and three-parameter Weibull fitting. The procedure of the proposed technique is proposed in Section 3. In Section 4, the proposed method is verified and the results are discussed. Finally, the conclusion of this paper is given in Section 5.

\section{Basic Theory}

2.1. Mathematical Morphological Fractal Dimension. Fractal dimension is able to describe the complexity of fractal object quantitatively, among which fractal box dimension is most widely used. The commonly used fractal box dimension has some disadvantages in accuracy [19], while mathematical morphological fractal dimension [20] replaces meshing coverage used in fractal box dimension with one-dimensional morphological coverage; thus, the calculation result is accurate and the calculation speed is fast which is suitable for online analysis. The calculation method is described as follows:

Assuming that time sequence is $f(n), n=1,2, \ldots, N$, the unit structuring element is $g$; according to the definition of multiscale morphology [21], structuring element under scale $\lambda$ is as follows:

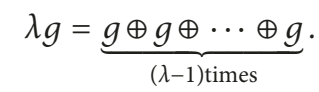

Define one-dimensional morphological coverage under scale $\lambda$ as follows:

$$
A_{g}(\lambda)=\sum_{n=1}^{N}((f \oplus \lambda g)(n)-(f \Theta \lambda g)(n))
$$

$A_{g}(\lambda)$ is able to meet the following conditions:

$$
\ln \left(\frac{A_{g}(\lambda)}{\lambda^{2}}\right)=D_{M} \ln \left(\frac{1}{\lambda}\right)+c, \quad\left(\lambda=1,2, \ldots, \lambda_{\max }\right),
$$

where $D_{M}$ is the mathematical morphological fractal dimension of the signal, $c$ is a constant, and $\lambda_{\max }$ is the largest analytical scale. The least squares linear fitting of $\ln \left(A_{g}(\lambda) / \lambda^{2}\right)$ and $\ln (1 / \lambda)$ is able to obtain the mathematical morphological fractal dimension.

2.2. Three-Parameter Weibull Fitting. Distribution function $F(t)$ of three-parameter Weibull distribution is as follows [22]: 


$$
F(t)=1-\exp \left[-\left(\frac{t-\gamma}{\eta}\right)^{m}\right] .
$$

Its distribution density function $f(t)$ is as follows:

$$
f(t)=\frac{m}{\eta}\left(\frac{t-\gamma}{\eta}\right)^{m-1} \exp \left[-\left(\frac{t-\gamma}{\eta}\right)^{m}\right]
$$

where $\eta$ is the scale parameter, $m$ is the shape parameter, and $\gamma$ means the position parameter. The parameter estimation methods include graphic method, moment estimation, linear regression estimation, and maximum likelihood method. In this paper, the maximum likelihood method is introduced to estimate the three parameters [23]. The main idea of which is regarding scale, shape parameter, and the maximum likelihood function as a function of the position parameter, obtaining parameters' value by means of traversing position parameter when the maximum likelihood value is obtained.

\section{Degradation Feature Extraction Flow}

An online degradation feature extraction technique based on morphological fractal dimension and sliding window Weibull fitting is proposed. The flowchart is shown in Figure 2.

(1) Vibration energy spectrum monitoring and acquisition: taking the online signal monitoring and acquisition system as the platform, vibration energy spectrum time sequence from the key measuring points of the shore bridge lifting mechanism gearbox is obtained.

(2) Degradation feature extraction using mathematical morphological fractal analysis. Setting an analysis period $T$ and proceeding mathematical morphological fractal analysis on the vibration energy spectrum within this period, morphological fractal dimension as the indicator is obtained and this parameter as the fractal feature for this period of sequence is taken. A continuous fractal curve will be obtained by means of repetitive operation.

(3) The fractal curve is divided into number of sliding windows; the width of sliding windows is set as $W$, and the intervals among the sliding windows are set as $S$.

(4) Proceeding three-parameter Weibull fitting within each sliding window, the scale parameter of each distribution which will be taken as the performance degradation feature of the sliding window is obtained.

(5) Taking online analysis and repeating steps (2)-(4), a scale parameter evolution curve will be obtained as performance degradation feature for the gearbox.

\section{Instance Data Analysis}

4.1. Shore Bridge Gearbox Full-Life Vibration Energy Spectrum. The full-life data of the hoisting mechanism gearbox obtained by the long-term monitoring from the

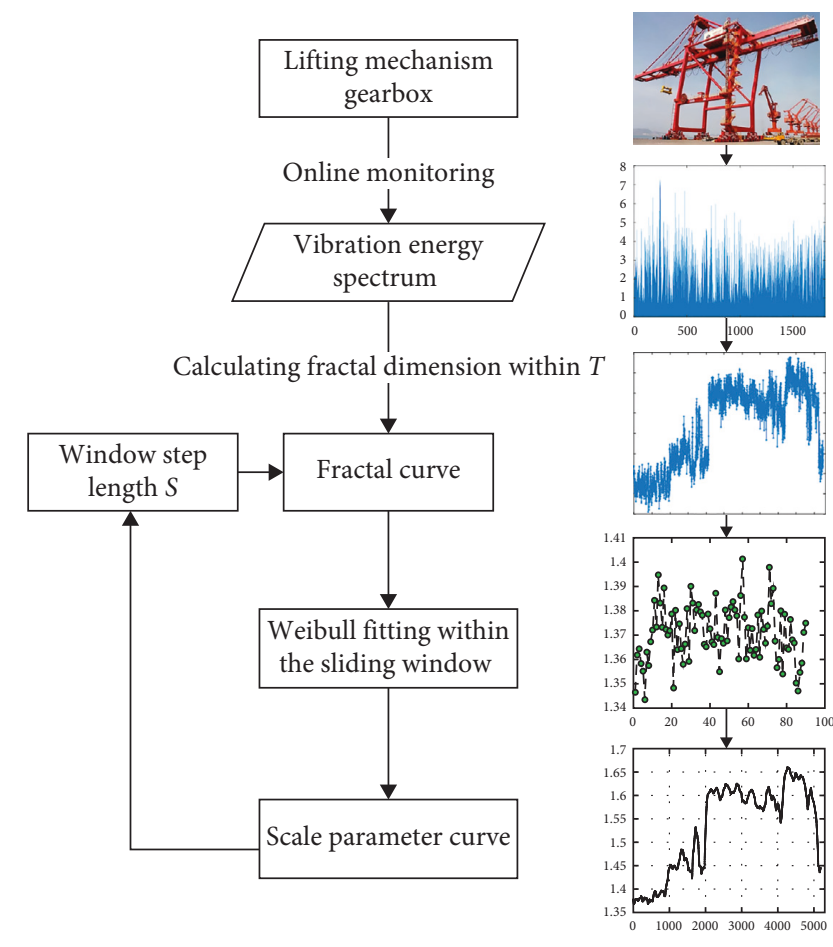

Figure 2: Online extraction process of degradation feature.

NetCMAS system are introduced for instance analysis. The shore bridge comes from a container terminal in Shanghai Port. The NetCMAS system had been working online for continuous monitoring since the shore bridge installation. The monitoring information includes vibration, temperature, and stress signals from more than 40 measuring points. The full-life vibration energy spectrum used in this paper comes from the vertical direction of the high-speed input shaft of the hoisting mechanism gearbox. The measurement point diagram is shown in Figure 3(a). The sampling frequency is $24 \mathrm{kHz}$, the sampling time is $1 \mathrm{~s}$, and the sampling interval is $10 \mathrm{~s}$. The effective value of the collecting data is calculated online by the on-site monitoring acquisition unit shown in Figure 3(b), forming an online vibration energy spectrum time sequence.

Failure occurred on the gearbox of the hoisting mechanism after nearly 7 years and 8 months of continuous monitoring. It was found that the failure position was the high-speed input shaft roller bearing of the gearbox after shutdown and dismounting, and the failure mode is running pulley abrasion. The on-site inspection map is shown in Figure 4.

Data cleaning is processed first to filter the vibration signals with low-value density collected in nonworking conditions; a processed time-domain waveform of the fulllife vibration energy spectrum sequence is shown in Figure 5. It is clear that the vibration energy of the measuring point is increasing with many occasional energy shocks, which were induced by the large impact caused by special container lifting operations. It is difficult to judge the timing of failure through the effective value accurately, and the occasional energy impact is likely to cause misjudgment; it is necessary for further degradation feature extraction. 


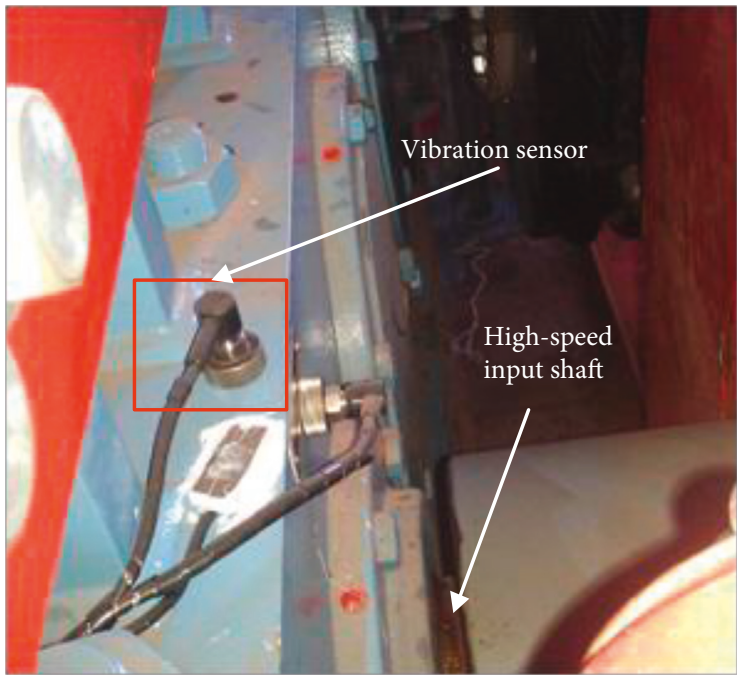

(a)

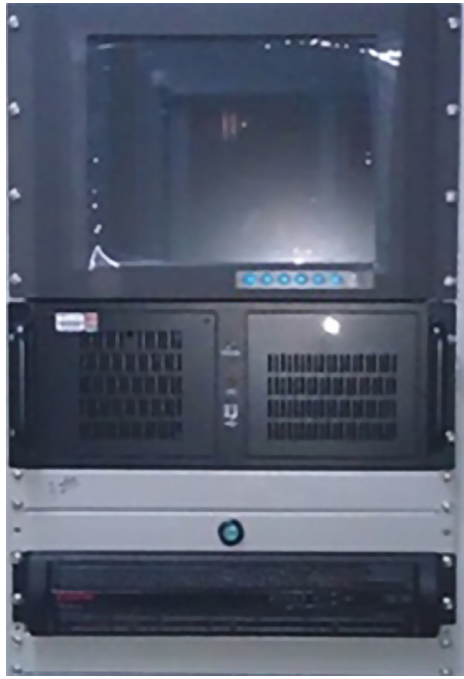

(b)

FIgURE 3: Data monitoring and collecting. (a) Vibration sensor on gear. (b) Monitoring and acquisition unit.

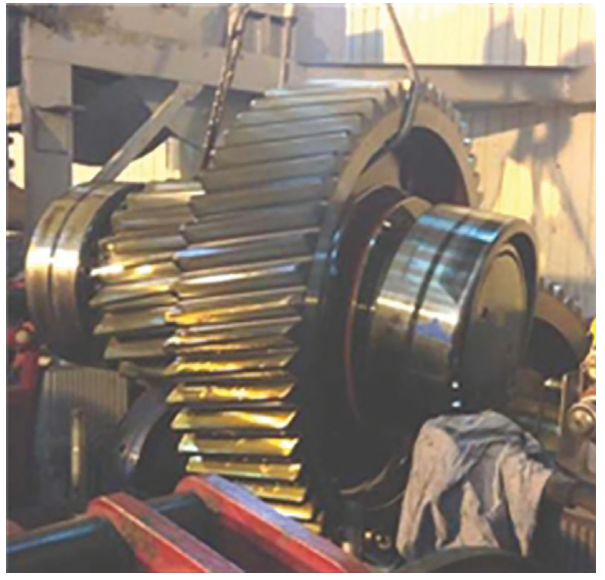

(a)

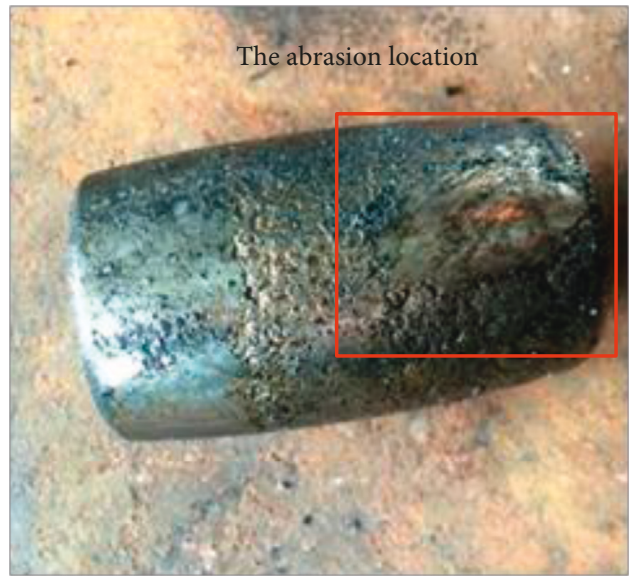

(b)

FIGURE 4: The on-site inspection map for gearbox bearing failure. (a) Dismounting of the hoisting mechanism gearbox. (b) Bearing pulley abrasion.

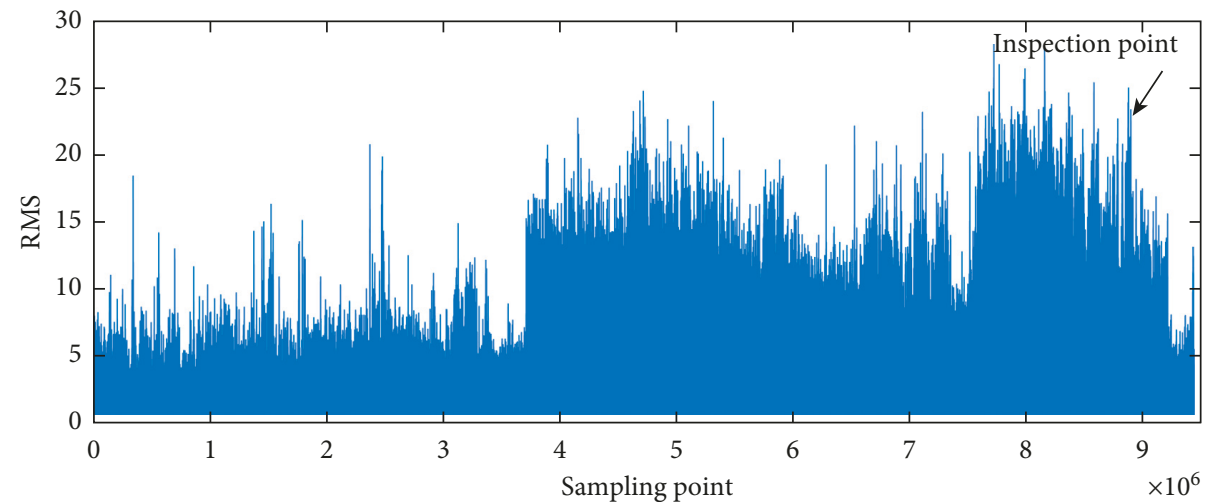

FIGURE 5: Time-domain waveform of the full-life vibration energy spectrum sequence. 


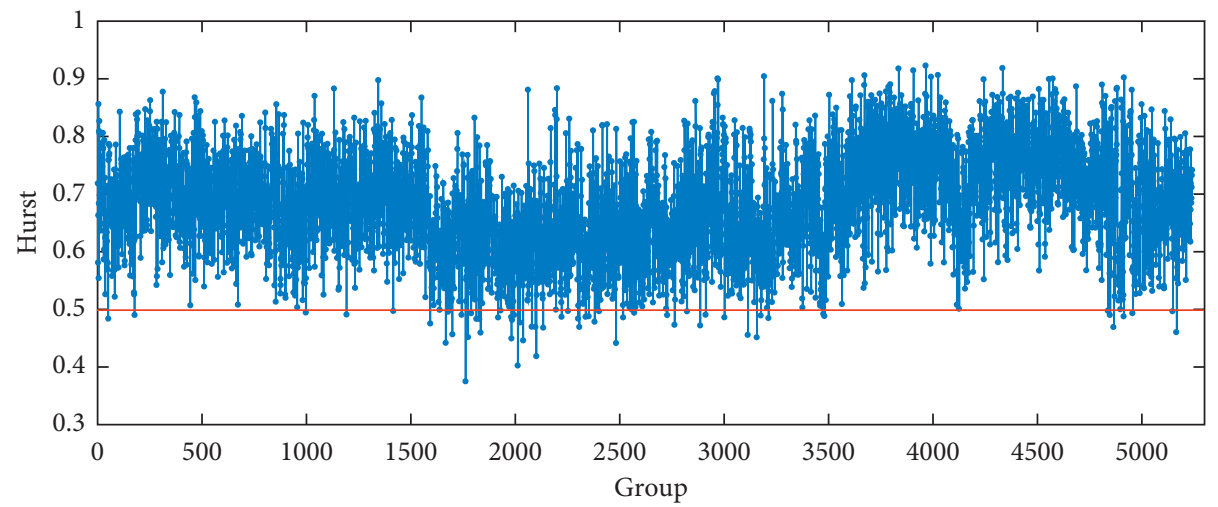

FIgURE 6: The Hurst curve evolution trend.

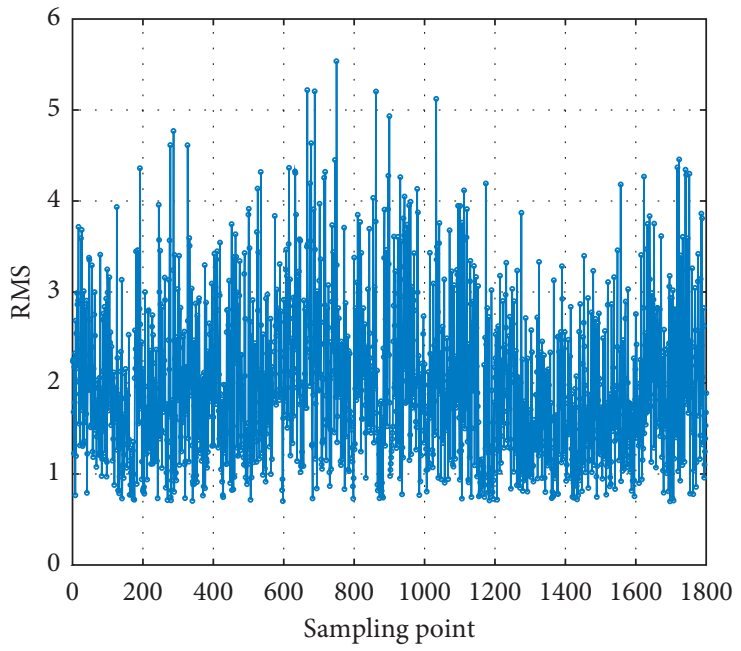

(a)

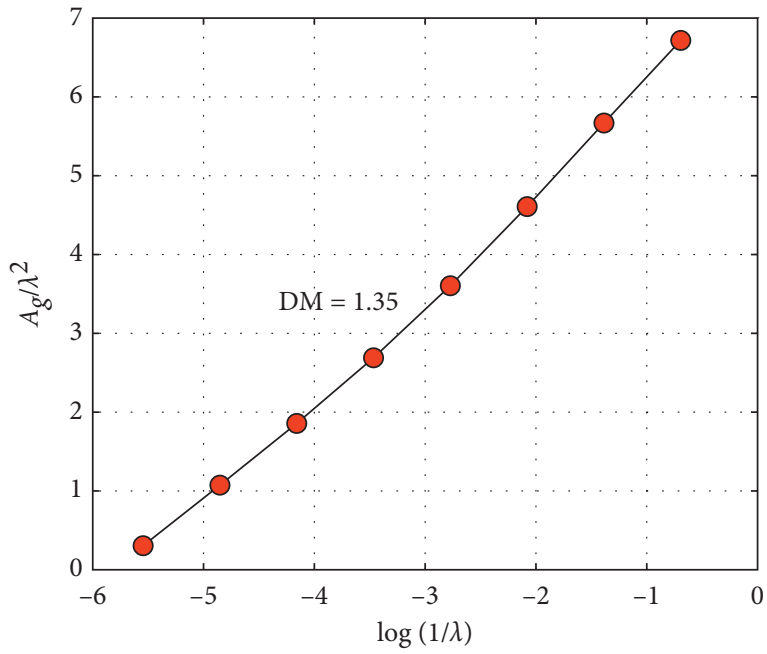

(b)

Figure 7: The calculation of morphological fractal dimension. (a) Energy spectrum in the analytical period. (b) Least squares linearity on morphological coverage and scale.

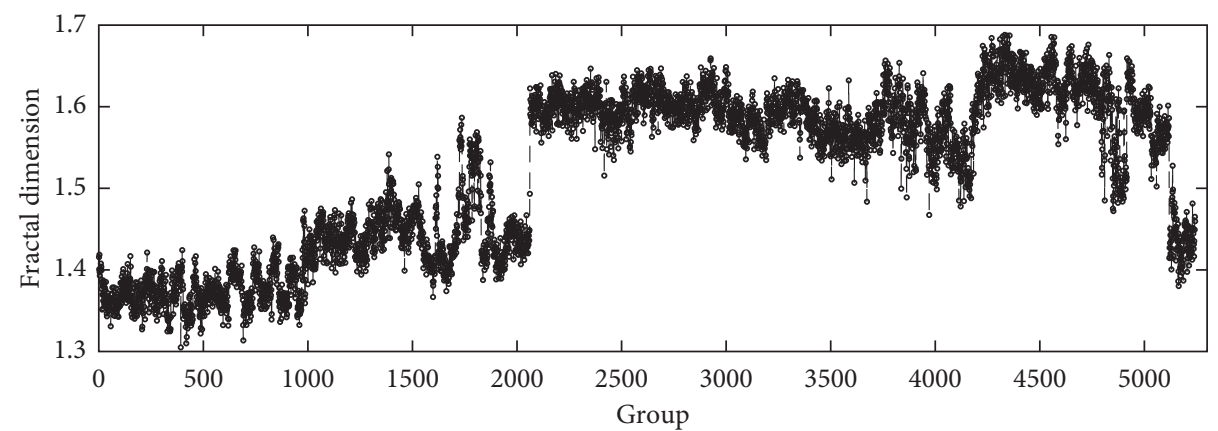

Figure 8: The fractal dimension evolution curve.

4.2. Degration Feature Extraction. Setting the analysis period as $T=1800$, the lifetime dataset is divided into 5243 groups. The full-life dataset is divided into 5243 groups.

In order to analyze the long-range correlation character of the energy spectrum sequence, Hurst indicator of each group is calculated by means of the $R / S$ method firstly [24]; the Hurst curve is shown in Figure 6. It can be seen that the Hurst indicator is greater than 0.5 in most groups, which indicates that the vibration energy spectrum signal of the gearbox has obvious long-range correlation character and it is effective to analyze the feature based on fractal theory.

Mathematical morphological fractal dimension is calculated for each group. Considering that energy-spectrum signal contains a large number of random impact components and triangular or rectangular structural elements are able to fit the impact character, triangular unit structuring 


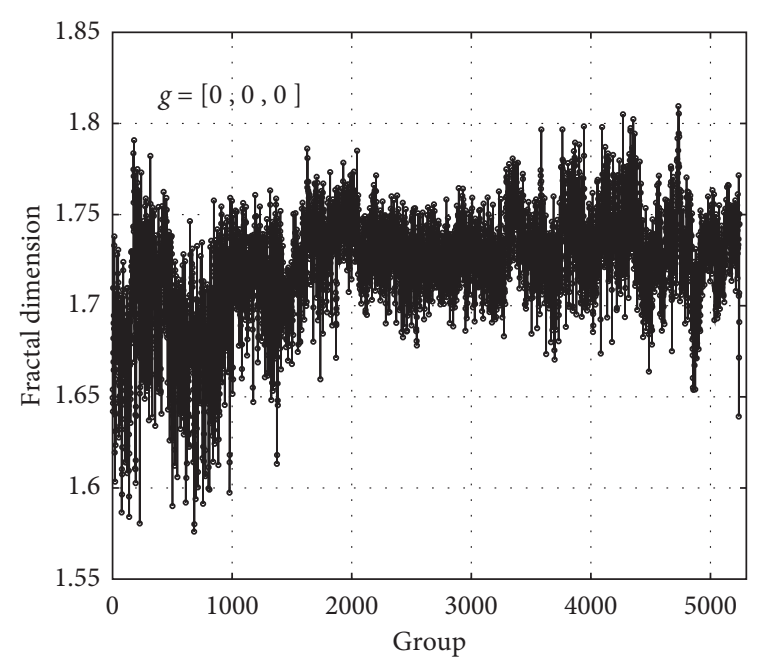

(a)

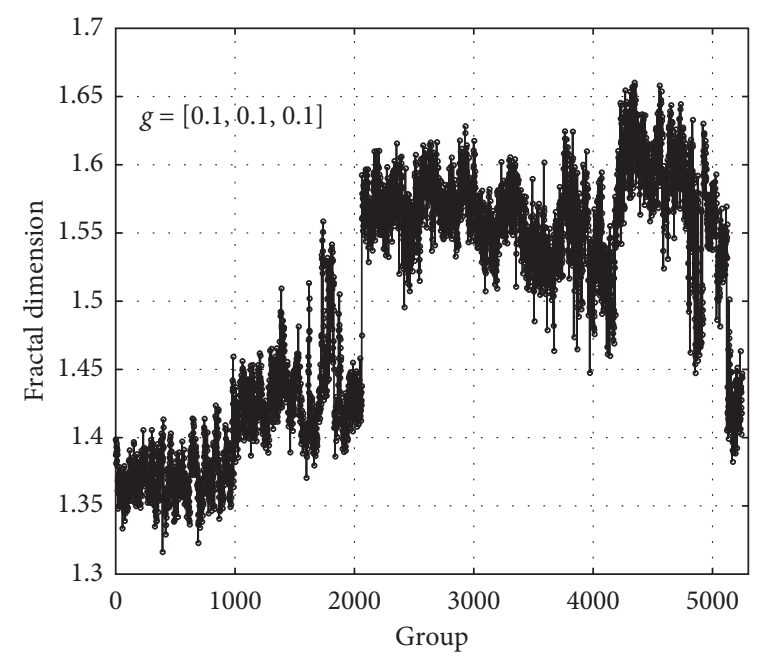

(b)

FIgURE 9: The fractal curves using different unit structuring elements (a) $g=[0,0,0]$ and (b) $g=[0.1,0.1,0.1]$.

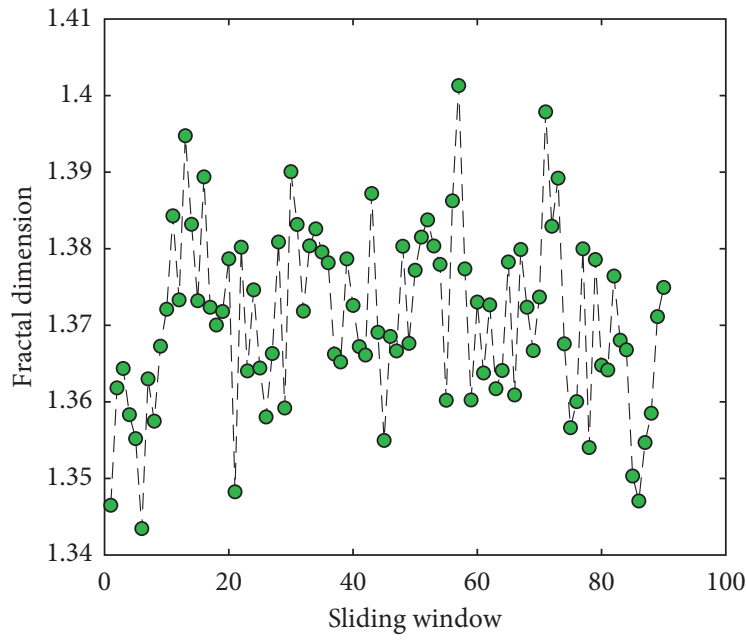

(a)

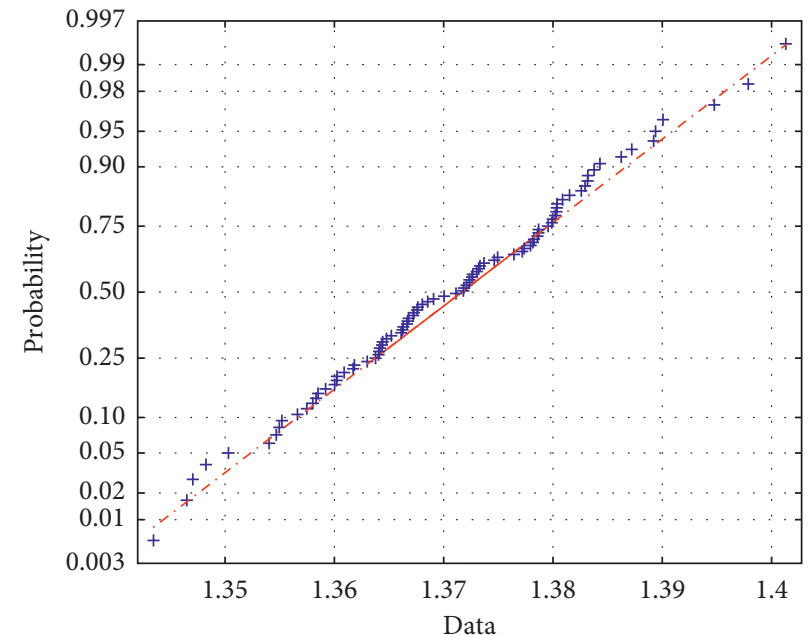

(b)

FIgURe 10: Sliding window Weibull distribution. (a) Sliding window fractal dimension sequence. (b) Weibull distribution of the sliding window.

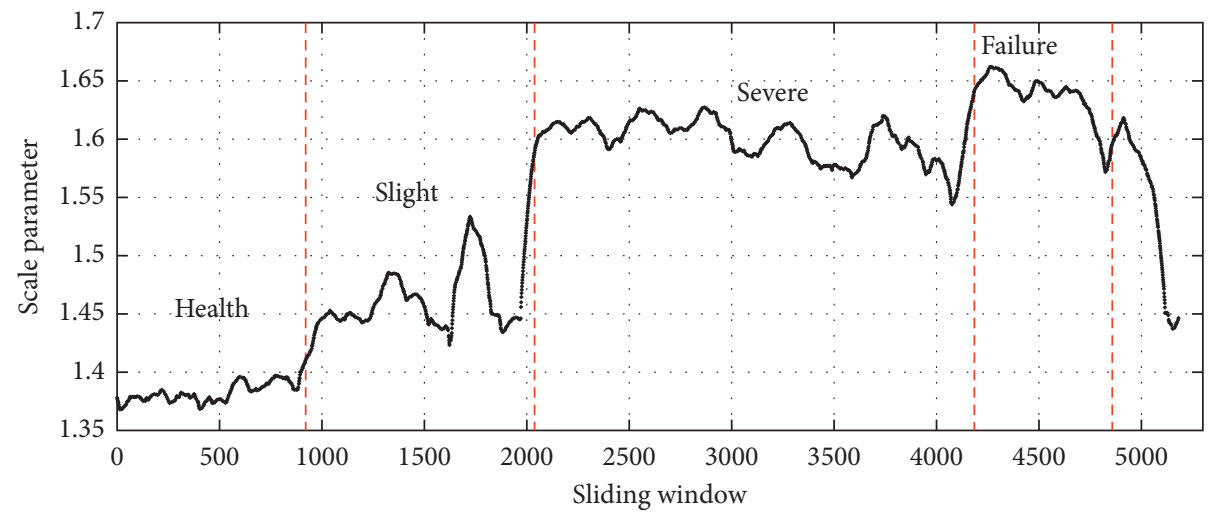

Figure 11: The evolution curve of the scale parameter. 


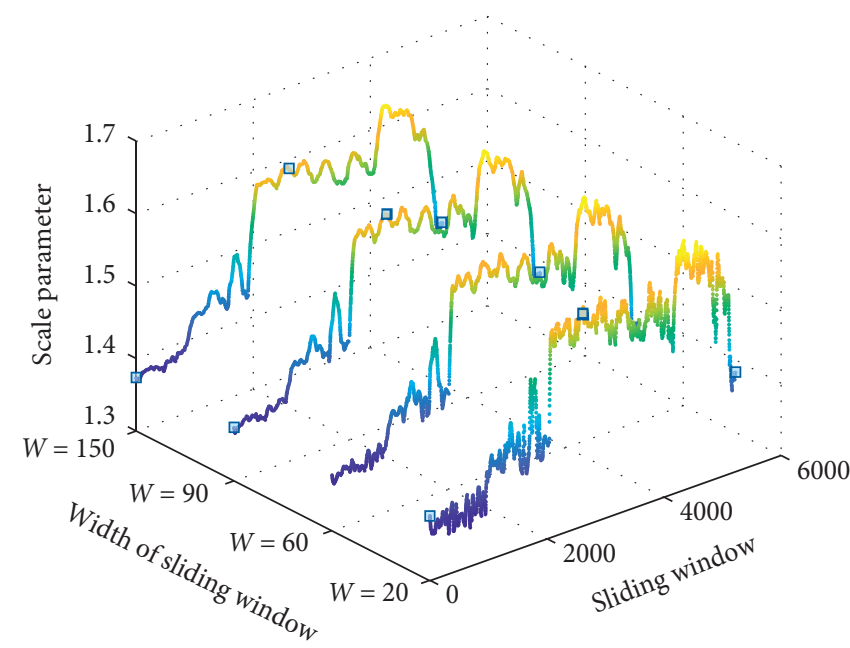

(a)

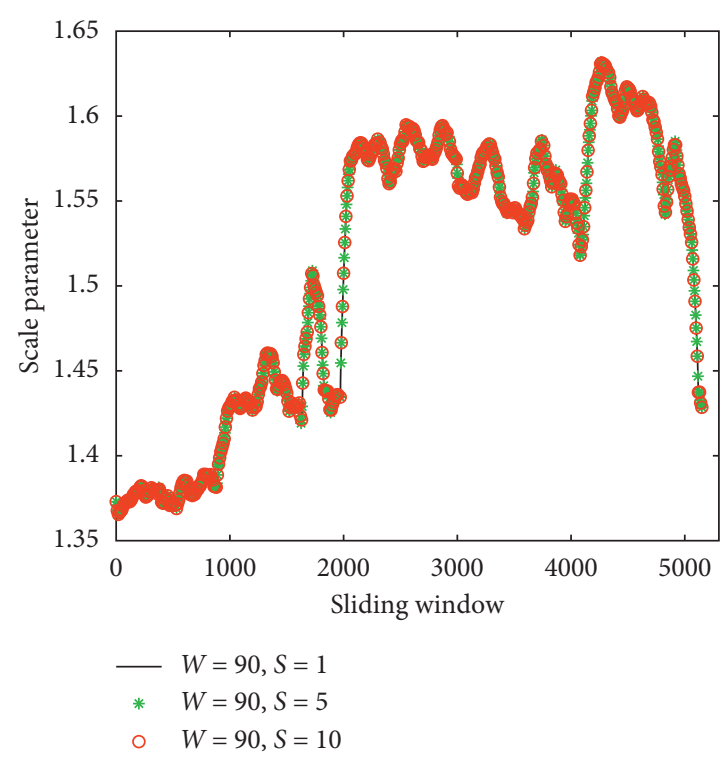

(b)

FIGURE 12: Analysis on the influence of different parameters. Comparison of (a) different sliding window's widths and (b) different sliding window's step size.

element $g=[0,0.1,0]$ is used and the analytical scale is set as $[2,4,8,16,32,64]$. Taking vibration energy spectrum in any group as the example, the wave curve is shown in Figure 7 (a). Calculation of mathematical morphological coverage and process least squares linearity on $\ln \left(A_{g}(\lambda) / \lambda^{2}\right)$ and $\ln (1 / \lambda)$ is shown in Figure 7(b).

The calculated fractal dimension evolution curve of the full-life dataset is shown in Figure 8. With the deepening of the degradation degree, the curve shows a main increasing trend with some random fluctuations due to random shocks.

Fractal dimension evolution curves using different unit structuring elements are shown in Figure 9. It can be seen that the curve calculated by flat unit structuring element $g=$ $[0,0,0]$ has a poor trend and does not reflect the trend information of the degradation process. A good result can also be obtained by using the rectangular unit structuring element $g=[0.1,0.1,0.1]$.

4.3. Sliding Window Weibull Fitting. The width of sliding window as $W=90$ and step size as $S=1$ are set. Three-parameter Weibull distribution is used to fit and obtain the shape parameter. The window in every $S$ step is translated and a repetitive execution of fitting is performed to obtain the shape parameter evolution curve. Morphological fractal dimension curve in any sliding window is shown in Figure 10(a), and statistical law is verified by Weibull distribution; the result is shown in Figure 10(b). It is clear that statistical probability of the window is approximately linear, which is consistent with the Weibull distribution.

An evolution curve of the scale parameter is obtained which is shown in Figure 11. It can be seen that as the degradation degree deepens, the curve has a whole increasing trend. Combined with practical experience, the entire degradation process can be approximated into four conditions including healthy, slight degradation, severe degradation, and a failure condition. After the maintenance and replacement of the bearing, the gearbox gradually transitions from the failure condition to the slight degradation condition after a running-in period, which is consistent with the actual inspection report of the port.

The scale parameter curves with different window's widths and step lengths are shown in Figure 11. It can be seen from Figure 12(a) that the smaller the value of window's width, the more variation details it will reflect. It is clear from Figure 12(b) that the step length does not change the value of the scale parameter and the smaller the step length, the higher of the evolving curve's accuracy. Therefore, it is possible to select an appropriate window width and step length in combination with industrial requirements.

In summary, through the morphological fractal feature extraction and sliding window Weibull fitting process, the scale parameter curve is able to clearly reflect the performance degradation pattern of the gearbox bearing contained in the industrial field data, and at the same time, the random impact is able to be filtered out effectively.

\section{Conclusion}

According to the character of shore bridge and its operating conditions, an online degradation feature extraction technique based on morphological fractal dimension and sliding window Weibull fitting is proposed. The full-life data of hoisting mechanism gearbox obtained by the long-term monitoring from the NetCMAS system are introduced for instance analysis, and the following conclusions are obtained.

(1) As to the commonly used bearing full-life acceleration test data, RMS is able to reflect the degradation 
degree of rolling bearing performance easily and has good condition tracking effect. For the field data of shore bridge, the vibration energy spectrum sequence fluctuates greatly and the random impact is obvious. It is difficult to accurately track the performance degradation pattern, which fully reflects the working conditions and data character of the shore bridge.

(2) Mathematical morphological fractal dimension is able to describe the fractal complexity of the vibration energy spectrum and has monotony character of the whole trend. As the degree of degradation continues to deepen, the value of fractal dimension also increases, reflecting the fractal evolution of the vibration load spectrum at the industrial site.

(3) The scale parameter of three-parameter Weibull distribution is able to reflect the performance degradation trend of the fractal curve smoothly, and it is effective for online degradation character of shore bridge gearbox. How to divide the health conditions of shore bridge will be the focus of the proceeding study.

\section{Data Availability}

The data used to support the findings of this study are available from the corresponding author upon request.

\section{Conflicts of Interest}

The authors declare no conflicts of interest in preparing this article.

\section{Acknowledgments}

This research received specific grant from the National High Technology Research Development Plan (2013AA041106), National Natural Science Foundation (31300783), and China Postdoctoral Science Foundation (2014M561458).

\section{References}

[1] D. Cristina and B. Paul, "Case study on use of vibrations diagnosis in exploitation of quay cranes," Constanta Maritime University Annals, vol. 14, no. 20, pp. 71-73, 2013.

[2] R. Araneo, G. Attolini, S. Celozzi, and G. Lovat, "Time-domain shielding performance of enclosures: a comparison of different global approaches," IEEE Transactions on Electromagnetic Compatibility, vol. 58, no. 2, pp. 434-441, 2016.

[3] R. B. Randall and J. Antoni, "Rolling element bearing diagnostics-a tutorial," Mechanical Systems and Signal Processing, vol. 25, no. 2, pp. 485-520, 2011.

[4] Y. Gao, F. Villecco, M. Li, and W. Song, "Multi-scale permutation entropy based on improved LMD and HMM for rolling bearing diagnosis," Entropy, vol. 19, no. 4, pp. 176-182, 2017.

[5] W. Zhipeng, J. Limin, and Q. Yong, "Adaptive diagnosis for rotating machineries using information geometrical KernelELM based on VMD-SVD," Entropy, vol. 28, no. 1, pp. 73-79, 2018.
[6] N. Saif, P. Amalin, and A. Anita, "Entropy-based feature extraction and classification of vibroarthographic signal using complete ensemble empirical mode decomposition with adaptive noise," IET Science, Measurement \& Technology, vol. 12, no. 3, pp. 350-359, 2018.

[7] J. Guo, X. Yuan, and C. Han, "Sensor selection based on maximum entropy fuzzy clustering for target tracking in large-scale sensor networks," IET Signal Processing, vol. 11, no. 5, pp. 613-621, 2017.

[8] Y. Wu, P. Shang, and Y. Li, "Modified generalized multiscale sample entropy and surrogate data analysis for financial time series," Nonlinear Dynamics, vol. 92, no. 3, pp. 335-1350, 2018.

[9] B. Wang, X. Hu, and H. Li, "Rolling bearing performance degradation condition recognition based on mathematical morphological fractal dimension and fuzzy C-means," Measurement, vol. 109, pp. 1-8, 2017.

[10] J. Zhang, C. W. Liu, F. R. Bi, X.-B. Bi, and X. Yang, "Fault feature extraction of diesel engine based on bispectrum image fractal dimension," Chinese Journal of Mechanical Engineering, vol. 31, no. 2, pp. 216-226, 2018.

[11] J. Lee, H. Qiu, G. Yu, and J. Lin, "Bearing dataset from IMS of University of Cincinnati and NASA ames prognostics data repository," 2007, http://ti.arc.nasa.gov/tech/dashpcoe/ prognostic-data-repository.

[12] P. Nectoux, Gourveaur, K. Medjaher et al., "PRONOSTIA: an experimental platform for bearings accelerated degradation tests," in Proceedings of the IEEE International Conference on Prognostics and Health Management, Denver, CO, USA, June 2012.

[13] D. Han, P. N. Zhao, and P. Shi, "Gear fault feature extraction and diagnosis method under different load excitation based on EMD, PSO-SVM and fractal box dimension," Journal of Mechanical Science and Technology, vol. 33, no. 2, pp. 487494, 2019.

[14] M. D. Prieto, A. G. Espinosa, J.-R. R. Ruiz, J. C. Urresty, and J. A. Ortega, "Feature extraction of demagnetization faults in permanent-magnet synchronous motors based on boxcounting fractal dimension," IEEE Transactions on Industrial Electronics, vol. 58, no. 5, pp. 1594-1605, 2011.

[15] B. Li, P. L. Zhang, G. Q. Ren, D.-S. Liu, and S.-S. Mi, "Mathematic morphology-based fractal dimension calculation and its application in fault diagnosis of roller bearings," Journal of Vibration \& Shock, vol. 29, no. 5, pp. 191-194, 2010.

[16] C. Chang, B. Tang, and Z. Lv, "Degradation trend prediction of rolling bearings based on Weibull distribution and least squares support vector machine," Journal of Vibration and Shock, vol. 33, no. 20, pp. 52-56, 2014.

[17] F.-T. Wang, C. Xu, C. Liu, H.-K. Li, Q.-K. Han, and H. Zhu, "Rolling bearing reliability assessment and life prediction based on KPCA and WPHM," Journal of Vibration,Measurement \& Diagnosis, vol. 37, no. 3, pp. 476-483+626, 2017.

[18] T. Gang, L. Jianxia, and H. Xiong, "Accurate approximate clustering algorithm and its application in the state monitoring of crane," Journal of Donghua University, vol. 44, no. 4, pp. 590-594, 2018.

[19] B. B. Chaudhuri and N. Sarkar, "Texture segmentation using fractal dimension," IEEE Transactions on Pattern Analysis and Machine Intelligence, vol. 17, no. 1, pp. 72-77, 1995.

[20] P. Maragos and F.-K. Sun, "Measuring the fractal dimension of signals: morphological covers and iterative optimization," IEEE Transactions on Signal Processing, vol. 41, no. 1, pp. 108-121, 1993.

[21] D. Feiyue, Y. Shao-pu, and G. Wen-wu, "Fault feature extraction method for rolling bearing based on adaptive multi- 
scale morphological AVG-Hat Filtering," Journal of Vibration Engineering, vol. 30, no. 6, pp. 1056-1065, 2017.

[22] R. Li, S. Zheng, Z. Wang, Y. Tang, S. Sun, and Z. Huang, "Evaluation of GNSS signal-in-space continuity: a Weibulldistribution-based method," Chinese Journal of Electronics, vol. 27, no. 3, pp. 634-640, 2018.

[23] S. Sel, M. Jung, and Y. Chung, "Bayesian and maximum likelihood estimations from parameters of McDonald extended Weibull model based on progressive type-II censoring," Journal of Statistical Theory and Practice, vol. 12, no. 2, pp. 1-24, 2017.

[24] D. Chunhong, W. Xueye, and L. Jinshan, "Fault diagnosis of gearboxes using nonlinearity and determinism by generalized Hurst exponents of shuffle and surrogate data," Entropy, vol. 20, no. 5, pp. 364-369, 2018. 


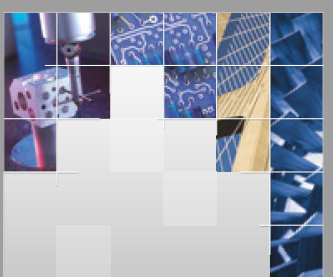

\section{Enfincering}
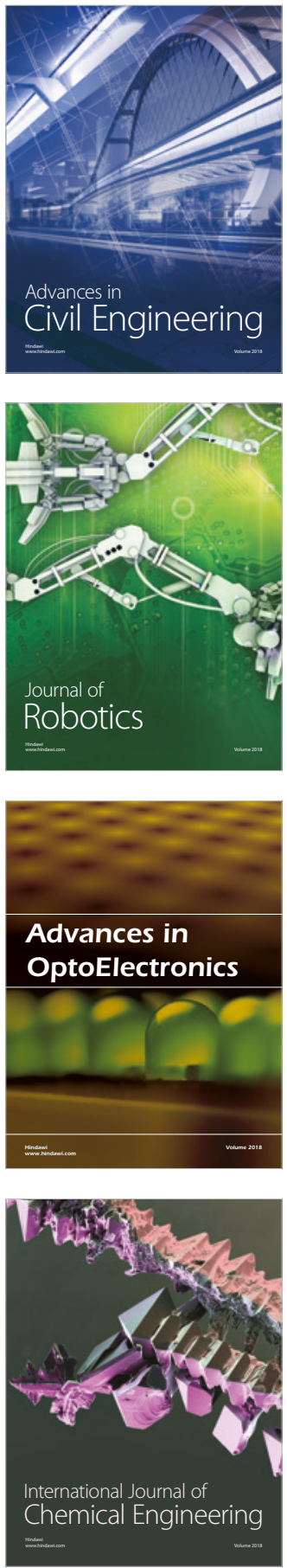

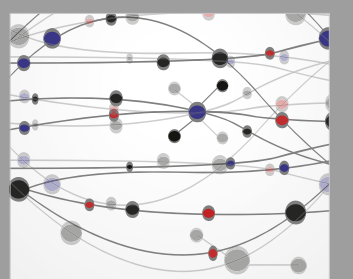

\section{Rotating \\ Machinery}

The Scientific World Journal

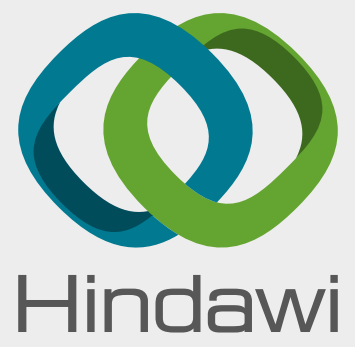

Submit your manuscripts at

www.hindawi.com
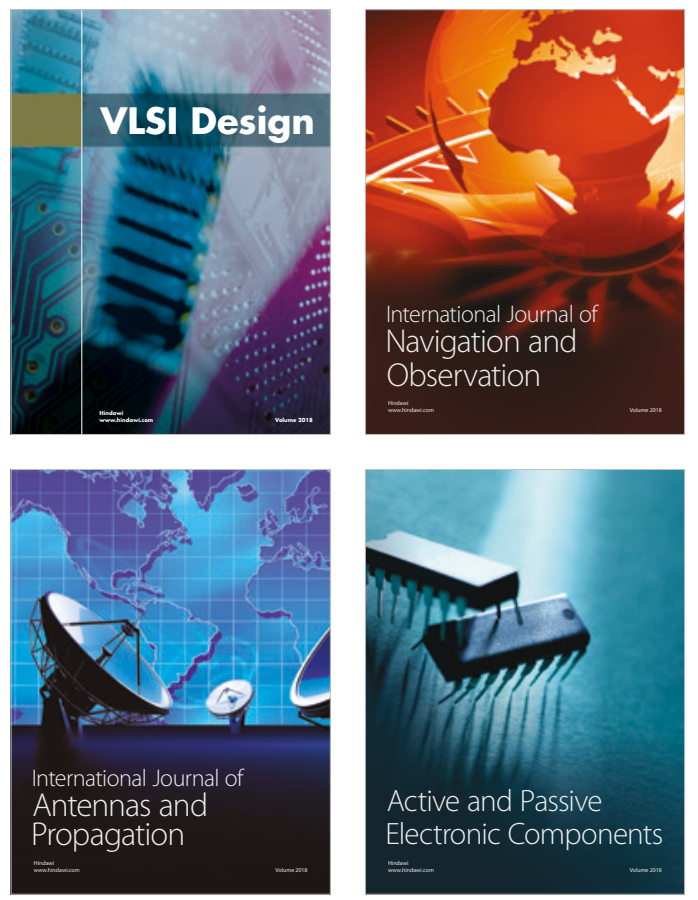
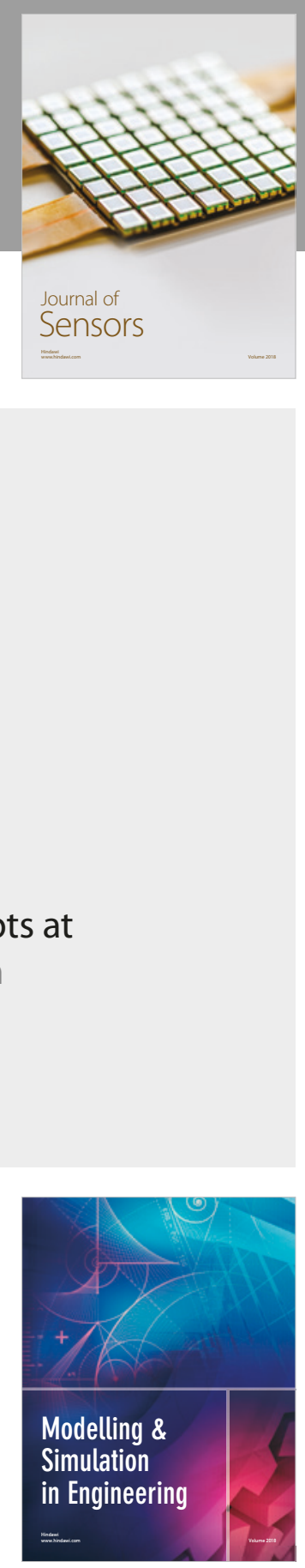

\section{Advances \\ Multimedia}
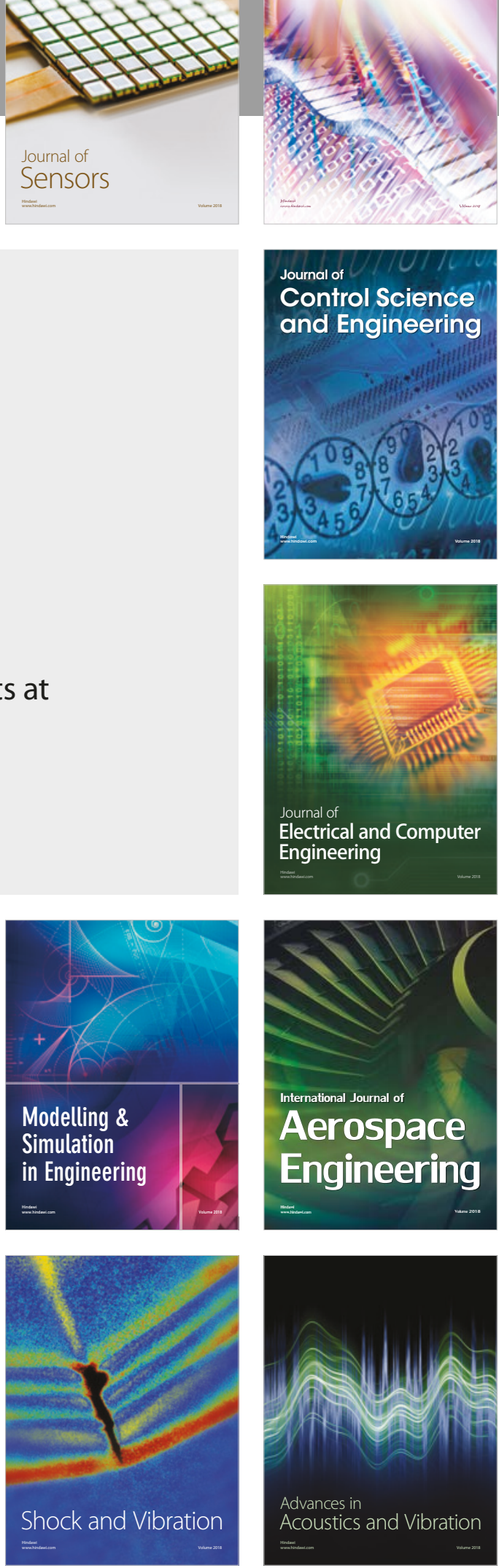